\title{
CONJUGACY SEPARABILITY OF CERTAIN 1-RELATOR GROUPS
}

\author{
C. Y. TANG ${ }^{1}$
}

\begin{abstract}
Let $G=\left\langle a, b ;\left(a^{l} b^{m}\right)^{t}\right\rangle$ where $l, m, t$ are integers. We show that groups of this type are conjugacy separable.
\end{abstract}

1. Introduction. The problem of conjugacy separability (c.s.) of groups was first raised by $\mathrm{A}$. W. Mostowski [13]. This is related to the conjugacy problem for groups since the solvability of the c.s. problem implies the solvability of the conjugacy problem. The conjugacy problem for generalized free products (g.f.p.) of two free groups with cyclic amalgamation was first solved by S. Lipschutz [11]. Later P. Stebe [15] partially solved the c.s. problem for g.f.p. of two free groups with cyclic amalgamation. Recently J. L. Dyer [9] showed that g.f.p. of two free groups with cyclic amalgamation are indeed c.s. In the same paper J. L. Dyer showed that 1-relator groups with nontrivial centers are c.s. (This result was also obtained by $\mathrm{S}$. M. Armstrong [4].) Thus, in particular, the fundamental group of a torus knot, $\langle a, b$; $\left.a^{l} b^{m}\right\rangle$, is c.s. The well-known Baumslag-Solitar group [7] shows that not all 1-relator groups are c.s. since this group is not even residually finite $\left({ }_{R} \mathcal{F}\right)$. However in [6] G. Baumslag conjectured that all 1-relator groups with nontrivial torsion are ${ }_{R} \mathscr{F}$. The difficulty of proving this conjecture is well known. Partial results are obtained in [1, $2,3,5]$. This led us to ask which 1-relator groups with nontrivial torsion are c.s.? In this paper we show that groups of the form $\left\langle a, b ;\left(a^{l} b^{m}\right)^{t}\right\rangle$ are c.s. This is because these groups are g.f.p. with cyclic amalgamation of two special types of c.s. groups. The proof is based on Solitar's theorem [12] and a modified approach of Stebe [15] using a more recent technique developed in [2]. In view of Baumslag's conjecture and results in $[3,5]$, it would be extremely interesting to see whether $G=$ $\left.\left\langle a, b ;\left(a^{-1} b^{l} a b^{m}\right)^{t}\right\rangle, t\right\rangle 1$, are c.s. Unfortunately it is still beyond the reach of our present technique. On the other hand the conjugacy problem for 1-relator groups with torsion is always solvable [14]. This makes the c.s. problem for 1-relator groups with torsion even more interesting.

2. Preliminaries. We shall make use of the following notations and terms:

$x \sim y$ means that $x, y$ are conjugates in a group.

$N \triangleleft_{f} G$ means that $N$ is a normal subgroup of finite index in $G$.

Received by the editors October 19, 1981 and, in revised form, February 25, 1982.

1980 Mathematics Subject Classification. Primary 20E25, 20F05; Secondary 20E30.

Key words and phrases. Conjugacy separable, conjugacy distinguishable, residually finite, generalized free product, potent, $H$-separable, M. Hall groups.

${ }^{1}$ The author gratefully acknowledges the partial support by the National Science and Engineering Research Council of Canada, Grant No. A-6064. 
$x$ is c.d. (conjugacy distinguishable) in $G$ means that if $y \in G$ and $x \nsim y$ then there exists $N \triangleleft_{f} G$ such that $x N \nsim y N$ in $G / N$. If every element of $G$ is c.d. then we say $G$ is c.s. (conjugacy separable).

Definition 2.1 [2]. Let $G$ be a group and $x \in G$. Then $G$ is said to be $\langle x\rangle$-potent, briefly $\langle x\rangle$-Pot, if, for every positive integer $n$, there exists $N \triangleleft_{f} G$ such that $x N$ has order exactly $n$ in $G / N$. $G$ is said to be potent if $G$ is $\langle x\rangle$-Pot for all $1 \neq x \in G$. The class of all potent groups shall be denoted by Pot.

Definition 2.2. Let $H$ be a subgroup of a group $G$. Then $G$ is said to be $H$-separable if, for all $x \in G \backslash H$, there exists $N \triangleleft_{f} G$ such that $x N \notin H N / N$ in $G / N$.

As a consequence of Solitar's theorem [12, p. 212], if $G=A{ }_{H} B$ and $x=x_{1} x_{2}$ $\cdots x_{n}, y=y_{1} y_{2} \cdots y_{n}$ are cyclically reduced words in $G$, each of minimal length in its conjugacy class, such that $x_{1}, y_{1} \in A$ (say) then $x \sim y$ in $G$ if and only if there exist $h_{0}, h_{1}, \ldots, h_{n} \in H$, such that $x_{i}=h_{i-1}^{-1} y_{i} h_{i}$, for $i=1,2, \ldots, n$ with $h_{0}=h_{n}$.

We also recall Lemmas 3.1, 3.2, 3.3 of [2].

Lemma 2.1. Let $G=A{ }{ }_{\langle h\rangle} B$, where $\langle h\rangle$ is infinite cyclic and $A, B$ are $\langle h\rangle$-separable and $\langle h\rangle$-Pot. If $K$ is a subgroup of $A$ and $A$ is $K$-separable then $G$ is $K$-separable.

Lemma 2.2. Let $G=A *{ }_{\langle h\rangle} B$, where $A$ is $\langle h\rangle$-Pot. If $x \in B$ and $B$ is $\langle x\rangle$-Pot, then $G$ is $\langle x\rangle$-Pot.

Lemma 2.3. The group $\left.M=\left\langle u, v ;(u v)^{t}\right\rangle, t\right\rangle 1$, is $\langle u\rangle$-separable, $\langle v\rangle$-separable and also $\langle u\rangle$-Pot and $\langle v\rangle$-Pot.

3. $G=\left\langle a, b ;\left(a^{l} b^{m}\right)^{t}\right\rangle$. In this section we shall make use of the g.f.p. of certain special groups with cyclic amalgamation to show that 1-relator groups of the type $G=\left\langle a, b ;\left(a^{l} b^{m}\right)^{t}\right\rangle$ are c.s. If $l$ or $m$ is zero it is easy to see that $G$ is c.s. Thus we can assume $m \neq 0$, say. We begin by proving some technical lemmas.

LEMMA 3.1. Let $\left.S=\left\langle b, d ;\left(d b^{m}\right)^{t}\right\rangle, m \neq 0, t\right\rangle 1$. Then $S$ is $\langle d\rangle$-separable and $\langle d\rangle$-Pot.

Proof. Let $M=\left\langle u, d ;(d u)^{t}\right\rangle$. By Lemma $2.3, M$ is $\langle d\rangle$-separable, and also $\langle u\rangle$-Pot and $\langle u\rangle$-separable. Now $S=M{ }^{*}{ }_{u=b^{m}}\langle b\rangle$. Therefore, by Lemma 2.1, $S$ is $\langle d\rangle$-separable. Since $M$ is also $\langle d\rangle$-Pot, by Lemma 2.3 , and $\langle b\rangle$ is $\left\langle b^{m}\right\rangle$-Pot, by Lemma 2.2, $S$ is $\langle d\rangle$-Pot.

LEMMA 3.2. Let $S=\left\langle b, d ;\left(d b^{m}\right)^{t}\right\rangle, m \neq 0, t>1$. If $s, w \in S$ such that $s \neq d^{\alpha} w d^{\beta}$ for all integers $\alpha$ and $\beta$ then there exists $N \triangleleft_{f} S$ such that $\bar{s} \neq \bar{d}^{\alpha} \bar{w} \bar{d}^{\beta}$ for all $\alpha$ and $\beta$, where $\bar{s}=s N, \bar{d}=d N$ and $\bar{w}=w N$.

Proof. Let $D=\langle d\rangle$. If $w \in D$ then, by Lemma 3.1, the lemma is true. Therefore we can assume $w \notin D$. Now $s \neq d^{\alpha} w d^{\beta}$ for all $\alpha, \beta$ implies that $w^{-1} s \neq w^{-1} d^{\alpha} w d^{\beta}$ for all $\alpha, \beta$. Since $S=\langle b\rangle *\left\langle c ; c^{t}\right\rangle$ with $d=c b^{-m}$ it follows that if $w \notin D$ then $\left[w, d^{\alpha}\right] \neq 1$ and $\left\langle w^{-1} d w, d\right\rangle$ is a free subgroup of rank 2 in $S$. Let $x=w^{-1} d w$ and $H=\langle x, d\rangle$. Now, by [8], $S$ is an M. Hall group. Therefore $H$ is a free factor of a subgroup $K$ of finite index in $S$. Let $K=L * H$. 
Case 1. $w^{-1} s \notin K$. Since $|S: K|<\infty$, it follows that there exists $N \triangleleft_{f} S$ such that $N \subseteq K$. Now $w^{-1} s \notin K$ implies $w^{-1} s \notin H$. In particular $w^{-1} s \neq x^{\alpha} d^{\beta}$ for all integers $\alpha, \beta$. Moreover $N \subseteq K$ implies that $\bar{w}^{-1} \bar{s} \neq \bar{x}^{\alpha} \bar{d}^{\beta}$, for all $\alpha, \beta$, in $G / N$, whence $\bar{s} \neq \bar{d}^{\alpha} \bar{w} \bar{d}^{\beta}$, for all $\alpha, \beta$, in $G / N$.

Case 2. $w^{-1} s \in K$ and $w^{-1} s \notin H$. Let $w^{-1} s=g_{1} h_{1} \cdots g_{n} h_{n}$ where $g_{i} \in L, h_{i} \in H$ and $g_{i}, h_{i} \neq 1$ except possibly for $g_{1}$ or $h_{n}$. Now, by [1], $S \in{ }_{R} \mathscr{F}$. Therefore $K \in{ }_{R} \mathscr{F}$ and $L \in{ }_{R} \mathcal{F}$. Thus there exists $\phi: K \rightarrow \hat{K}$ where $\hat{K}=L \phi * H \phi$ such that $L \phi$ and $H \phi$ are both finite with $g_{i} \phi \neq 1, h_{i} \phi \neq 1$. Now $w^{-1} s \notin H$. This implies that $\left(w^{-1} s\right) \phi \notin$ $H \phi$. Since $H \phi$ is finite and $\hat{K} \in{ }_{R} \mathscr{F}$, it follows that there exists $\psi: \hat{K} \rightarrow K^{\prime}$ such that $K^{\prime}$ is finite and $\left(w^{-1} s\right) \phi \psi \notin H \phi \psi$. Let $N=\operatorname{ker} \phi \psi$. Then $|S: N|<\infty$. Hence there exists $M \triangleleft_{f} S$ such that $\left(w^{-1} s\right) M \notin H M / M$. This implies $\bar{w}^{-1} \bar{s} \neq \bar{x}^{\alpha} \bar{d}^{\beta}$, for all integers $\alpha, \beta$, in $G / M$, whence $\bar{s} \neq \bar{d}^{\alpha} \bar{w} \bar{d}^{\beta}$, for all $\alpha, \beta$, in $G / M$.

Case 3. $w^{-1} s \in H$. Let $w^{-1} s=x^{\alpha_{1}} d^{\beta_{1}} \cdots x^{\alpha_{n}} d^{\beta_{n}}$ where $\alpha_{i}, \beta_{i}$ are not zero for all $i$, except possibly for $\alpha_{1}$ or $\beta_{n}$. Moreover, since $w^{-1} s \neq x^{\alpha} d^{\beta}$ for all integers $\alpha, \beta$ we can assume $n>1$. Now $K=L * H$ and $H \in{ }_{R} \mathscr{F}$. Thus it is not difficult to see that using a similar argument as in Case 2 , there exists $N \triangleleft_{f} S$ such that $\bar{s} \neq \bar{d}^{\alpha} \bar{w} \bar{d}^{\beta}$, for all integers $\alpha, \beta$, in $G / N$.

This completes the proof.

LEMMA 3.3. Let $G$ be a group and $g, h \in G$ such that $\left[h^{-1} g^{m} h, g^{m}\right] \neq 1$ where $|g|=m p^{t}, p$ a prime and $(m, p)=1$. If $h^{-1} g^{r} h=g^{s}$ then $p$ divides both $r$ and $s$.

Proof. Suppose $p \nmid r$. This means $\left(r, p^{t}\right)=1$. Therefore there exist integers $\alpha, \beta$ such that $\alpha r+\beta p^{t}=1$. This implies $\alpha m r+\beta m p^{t}=m$. It follows that $\left(h^{-1} g^{r} h\right)^{\alpha m}$ $=h^{-1} g^{m} h=g^{s \alpha m}$. But this implies that $\left[h^{-1} g^{m} h, g^{m}\right]=1$ contradicting the hypothesis that $\left[h^{-1} g^{m} h, g^{m}\right] \neq 1$. Hence $p \mid r$.

On the other hand if $p \nmid s$ then $\left(s, p^{t}\right)=1$. Thus there exist integers $\alpha, \beta$ such that $\alpha s+\beta p^{t}=1$, whence $\alpha m s+\beta m p^{t}=m$. It follows that

$$
\left(h^{-1} g^{m} h\right)^{\alpha r}=\left(h^{-1} g^{r} h\right)^{\alpha m}=g^{\alpha m s}=g^{m} \text {. }
$$

But this again implies that $\left[h^{-1} g^{m} h, g^{m}\right]=1$, contradicting the hypothesis of the lemma. Hence $p \mid s$.

Lemma 3.4. Let $\left.S=\left\langle b, d ;\left(d b^{m}\right)^{t}\right\rangle, m \neq 0, t\right\rangle 1$, and let $G=S{ }_{d=a^{\prime}}\langle a\rangle$. Let $x=x_{1} x_{2} \cdots x_{n}$ and $y=y_{1} y_{2} \cdots y_{n}, n>1$, be cyclically reduced words in $G$ such that $x \propto y$ in $G$. Suppose, for each $i$, there exist integers $\alpha_{i}, \beta_{i}$ such that $x_{i}=d^{-\alpha_{i}} y_{i} d^{\beta_{i}}$. Then there exists $\theta: G \rightarrow \hat{G}$ such that $\hat{G}$ is finite and $x \theta \nsim y \theta$ in $\hat{G}$.

Proof. WLOG we can assume $x_{1}, y_{1} \in A=\langle a\rangle$. Thus $x_{2 i}, y_{2 i} \in S$. By B. B. Newman [14], the centralizer $Z_{S}(d)$ of $d$ in $S$ is cyclic. In fact $Z_{S}(d)=\langle d\rangle=D$. Since $\left[x_{2 i-1}, d\right]=\left[y_{2 i-1}, d\right]=1$, WLOG we can let $x_{2 i-1}=y_{2 i-1}$ where $i=1, \ldots, k$ and $n=2 k$. Thus we can assume $\alpha_{2 i-1}=\beta_{2 i-1}=0$. Consider the differences $\Delta_{2}=\alpha_{2}-\beta_{2 k}, \Delta_{4}=\alpha_{4}-\beta_{2}, \ldots, \Delta_{2 k}=\alpha_{2 k}-\beta_{2(k-1)}$. If all the $\Delta_{2 i}$ are zero then $x \sim y$ in $G$ contradicting the assumption $x \nsim y$ in $G$. Hence at least one of the $\Delta_{2 i}$ is not zero. Let $p$ be a prime which does not divide at least one of the $\Delta_{2 i}$.

Next, consider $S=\left\langle d, b ;\left(d b^{m}\right)^{t}\right\rangle$. Since $d b^{m}$ is primitive in the free group $\langle d, b\rangle$, by Fischer, Karrass and Solitar [10], $S$ is free-by-finite. Let $F$ be a free normal 
subgroup of finite index in $S$ and let $|S: F|=p^{e} \lambda$ where $(p, \lambda)=1$. If $s \in S$ and $s \notin F$ then there exists an integer $f$ such that $f \mid p^{e} \lambda$ and $s^{f} \in F$.

By Lemma 3.1, $S$ is $D$-separable and $D$-Pot. Thus there exists $N_{1} \triangleleft_{f} S$ such that, for all $i=1, \ldots, k, \quad x_{2 i}, y_{2 i} \notin D N_{1}, \quad d^{\alpha_{2 i}}, \quad d^{\beta_{2 i}} \notin N_{1}$ and $\left[x_{2 i}^{-1} d^{g} x_{2 i}, d^{g}\right]$, $\left[y_{2 i}^{-1} d^{g} y_{2 i}, d^{g}\right] \notin D N_{1}$ for all $g \mid \lambda$. Since $F \triangleleft_{f} S$ and $F$ is free, whence a residually $p$-group, we can assume $\left|S / N_{1}\right|=p^{r_{1}} \lambda$. In the same way, since $A$ is both $D$-separable and $D$-Pot, there exists $N_{2} \triangleleft_{f} A$ such that $x_{2 i-1}, y_{2 i-1} \notin D N_{2}$ and that $\left|A / N_{2}\right|=$ $p^{r_{2}} \mu$. Moreover, by the $D$-potency of $S$ and $A, N_{1}$ and $N_{2}$ can be chosen such that $D \cap N_{1}=D \cap N_{2}$.

Let $\phi$ be the canonical map of $G$ onto $\bar{G}=\bar{S} *_{D}-\bar{A}$, where $\bar{S}=S / N_{1}, \bar{A}=A / N_{2}$ and $\bar{D}=D / N_{2} \cap D$. For convenience we shall use $\bar{z}$ to denote $z \phi$ for $z \in G$. Let the order of $\bar{d}$ be $p^{\alpha} \lambda^{\prime}$ where $\lambda^{\prime} \mid \lambda$. Now, in $\bar{G},\left[\bar{y}_{2 i}^{-1} \bar{d}^{\lambda^{\prime}} y_{2 i}, \bar{d}^{\lambda^{\prime}}\right] \neq 1$ and $\bar{x}_{2 i}=\bar{d}^{-\alpha_{2 i}} \bar{y}_{2 i} \bar{d}^{\beta_{2 i}}$. If also $\bar{x}_{2 i}=\bar{d}^{-\gamma_{2 i}} \bar{y}_{2 i} \bar{d}^{\delta_{2 i}}$ then

$$
\bar{y}_{2 i}^{-1} \bar{d}^{\alpha_{2 i}-\gamma_{2 i}} \bar{y}_{2 i}=\bar{d}^{-\left(\delta_{2 i}-\beta_{2 i}\right)} \text {. }
$$

Thus, by Lemma 3.3, $p \mid \alpha_{2 i}-\gamma_{2 i}$ and $p \mid \delta_{2 i}-\beta_{2 i}$. On the other hand $\left[\bar{x}_{2 i-1}, \bar{d}\right]=1$ implies that we can let $\gamma_{2 i-1}=\delta_{2 i-1}=0$. Hence $\alpha_{j} \equiv \gamma_{j}(p)$ and $\beta_{j} \equiv \delta_{j}(p)$ for all $j=1, \ldots, n$.

Now $\bar{x} \sim \bar{y}$ in $\bar{G}$ if and only if there exist integers $\gamma_{2 i}, \delta_{2 i}$ such that $\bar{x}_{2 i}=\bar{d}^{-\gamma_{2 i}} \bar{y}_{2 i} \bar{d}^{\delta_{2 i}}$ with $\bar{d}^{\gamma_{2}}=\bar{d}^{\delta_{2 k}}, \bar{d}^{\gamma_{4}}=\bar{d}^{\delta_{2}}, \ldots, \bar{d}^{\gamma_{2 k}}=\bar{d}^{\delta_{2(k-1)}}$. Since $p$ divides the order of $\bar{d}$, this means $p$ divides each of $\gamma_{2}-\delta_{2 k}, \gamma_{4}-\delta_{2}, \ldots, \gamma_{2 k}-\delta_{2(k-1)}$. It follows that $p$ divides each of the $\Delta_{2 i}$, contradicting the choice of $p$. Hence $\bar{x} \nsim \bar{y}$ in $\bar{G}$. Since $\bar{S}$ and $\bar{A}$ are both finite, by [9], $\bar{G}$ is c.s. Thus there exists $\psi: \bar{G} \rightarrow \hat{G}$ such that $\hat{G}$ is finite and $\bar{x} \psi \nsim \bar{y} \psi$ in $\hat{G}$. Let $\theta=\phi \psi$. Then $x \theta \nsim y \theta$ as required.

THEOREM 3.5. $G=\left\langle a, b ;\left(a^{l} b^{m}\right)^{t}\right\rangle$ is c.s.

Proof. If $l$ or $m=0$ then $G$ is a free product of cyclic groups, whence $G$ is c.s.

Thus in the following considerations we shall always assume $l, m \neq 0$. If $t=1$ then $G$ has a nontrivial center. Thus, by Armstrong [4] (or Dyer [9, Theorem 12]), $G$ is c.s. Hence we need only consider the case when $t>1$.

Let $S=\left\langle b, c ; c^{t}\right\rangle$. Clearly $S=\langle b\rangle *\left\langle c ; c^{t}\right\rangle$ is c.s. Let $d=c b^{-m}$. Then $S=$ $\left\langle b, d ;\left(d b^{m}\right)^{t}\right\rangle$. Further if we let $P=S{ }^{*}{ }_{d=a^{l}}\langle a\rangle$ then $P=\left\langle a, b ;\left(a^{l} b^{m}\right)^{t}\right\rangle=G$. We shall show that $P$ is c.s. By Lemma $3.1, S$ is $\langle d\rangle$-separable and $\langle d\rangle$-Pot. Since $\langle a\rangle=A$ is $\left\langle a^{l}\right\rangle$-Pot, i.e. $\langle d\rangle$-Pot, by Lemma $2.2, P$ is $\langle d\rangle$-Pot.

Now let $x, y \in P$ such that $x \nsim y$ in $P$. We can assume $x, y$ to be cyclically reduced as words on $S$ and $A$. Moreover we can assume $x, y$ to be of minimal lengths in their conjugacy classes. We use $\|z\|$ to denote the cyclically reduced length of $z \in P\left[12\right.$, p. 212]. If $\|x\| \neq\|y\|$, let $x=x_{1} \cdots x_{n}, y=y_{1} \cdots y_{m}, n \neq m$, be cyclically reduced. Since $S$ and $A$ are $\langle d\rangle$-separable and $\langle d\rangle$-Pot there exist $N \triangleleft_{f} S$ and $M \triangleleft_{f} A$ such that $N \cap D=M \cap D$ and $x_{i} N, y_{j} M \in \bar{S} \backslash \bar{D}$ for $x_{i}, y_{j} \in S \backslash D$ and $x_{i} N, y_{j} M \in \bar{A} \backslash \bar{D}$ for $x_{i}, y_{j} \in A \backslash D$, where $\bar{S}=\bar{S} / \bar{N}, \bar{A}=A / M$ and $\bar{D}=D / N$ $\cap D$. Let $\phi$ be the canonical homomorphism of $P$ onto $\bar{P}=\bar{S} * \bar{A}$. Then $\|x\|=$ $\|x \phi\| \neq\|y \phi\|=\|y\|$. Thus $\|x \phi\|$ and $\|y \phi\|$ are each of minimal lengths in their respective conjugacy classes with $x \phi \nsim y \phi$. Since $\bar{S}$ and $\bar{A}$ are finite, $\bar{P}$ is c.s. Hence 
$x, y$ are c.d. in $P$ if $\|x\| \neq\|y\|$. Thus we need only consider the cases when $\|x\|=\|y\|$.

Case 1. $\|x\|=\|y\|=0$. Since $S$ and $A$ are c.s., this implies that there exist $N_{1} \triangleleft_{f} S$ and $N_{2} \triangleleft_{f} A$ such that the respective images of $x$ and $y$ in $S / N_{1}$ and $A / N_{2}$ are not conjugates. Let $N_{1} \cap D=\left\langle d^{\alpha}\right\rangle$ and $N_{2} \cap D=\left\langle d^{\beta}\right\rangle$, where $D=\langle d\rangle$. Since $P$ is $\langle d\rangle$-Pot, there exists $M \triangleleft_{f} P$ such that $M \cap D=\left\langle d^{\alpha \beta}\right\rangle$. Let $M_{1}=M \cap N_{1}$ and $M_{2}=M \cap N_{2}$. Then, clearly, $M_{1} \cap D=M_{2} \cap D=\left\langle d^{\alpha \beta}\right\rangle$. It follows that $P$ can be mapped onto $\bar{P}=\bar{S}{ }_{*} \bar{D} \bar{A}$, where $\bar{S}=S / M_{1}, \bar{A}=A / M_{2}$ and $\bar{D}=D /\left\langle d^{\alpha \beta}\right\rangle$. Since $\bar{S}$ and $\bar{A}$ are finite, by Dyer [9, Theorem 1], $\bar{P}$ is c.s. Now, by the choice of $M_{1}$ and $M_{2}$, the images of $x$ and $y$ in $\bar{P}$ are not conjugates in $\bar{P}$. Hence $x$ and $y$ are c.d. in $P$.

Case 2. $\|x\|=\|y\|=1$. Suppose $x, y \in S$ or $x, y \in A$. Then by a similar argument as in Case 1 we can show that $x$ and $y$ are c.d. in $P$. Thus we can assume $x \in S$ and $y \in A$. Now any homomorphic image of $A$ is cyclic, whence the corresponding image of $y$ has only itself in its conjugacy class. Let $\theta$ be the canonical map of $P$ onto $\bar{P}=P / S^{P}$. Then $\bar{P}=\left\langle a \theta ;(a \theta)^{l}\right\rangle$. Since $\|y\|=1$, it follows that $y \theta \neq 1$. On the other hand $x \in S$ implies that $x \theta=1$. Hence $x$ and $y$ are c.d. in $P$.

Case 3. $\|x\|=\|y\| \geqslant 2$. We can assume that $x=x_{1} x_{2} \cdots x_{n}$ and $y=y_{1} y_{2} \cdots y_{n}$ are cyclically reduced words in $P$ such that $x_{1}, y_{1} \in A$ and $x_{2 i}, y_{2 i} \in S$ where $i=1,2, \ldots, k$ and $n=2 k$. If there exists an integer $j$ such that $x_{2 j} \notin D y_{2 j} D$, where $D=\langle d\rangle$, then, by Lemma 3.2, there exists $N \triangleleft_{f} S$ such that $\bar{x}_{2 j} \notin \bar{D}_{\bar{y}_{2 j}} \bar{D}$, where $\bar{x}_{2 j}=x_{2 j} N, \bar{y}_{2 j}=y_{2 j} N$ and $\bar{D}=D N / N$ in $S / N$. Now $S$ and $A$ are both $D$-separable. Thus there exist $N_{1} \triangleleft_{f} S$ such that $x_{2 i} \notin N_{1} D$ and $N_{2} \triangleleft_{f} A$ such that $x_{2 i-1} \notin$ $N_{2} D$ for all $i$. Since $S$ and $A$ are both $D$-Pot, there exist $M_{1} \triangleleft_{f} S$ and $M_{2} \triangleleft_{f} A$ such that $M_{1} \subseteq N \cap N_{1}, M_{2} \subseteq N_{2}$ and $M_{1} \cap D=M_{2} \cap D$. Let $\theta$ be the canonical homomorphism of $P$ onto $\hat{P}=\hat{S} * \hat{D} \hat{A}$ where $\hat{S}=S / M_{1}, \hat{A}=A / M_{2}$ and $\hat{D}=D / M_{1} \cap D$. Clearly $x_{2 j} \theta \notin \hat{D}\left(y_{2 j} \theta\right) \hat{D}$. Hence, by Solitar's theorem, $x \boldsymbol{\theta} \nsim y \theta$ in $\hat{P}$. Since $\hat{P}$ is c.s. it follows that $x$ and $y$ are c.d. in $P$. On the other hand if $x_{i} \in D y_{i} D$ for all $i$, then, by Lemma 3.4, again we have $x$ and $y$ to be c.d. in $P$.

This proves that $G$ is c.s.

\section{REFERENCES}

1. R. B. J. T. Allenby, L. E. Moser and C. Y. Tang, The residual finiteness of certain one-relator groups, Proc. Amer. Math. Soc. 78 (1980), 8-10.

2. R. B. J. T. Allenby and C. Y. Tang, The residual finiteness of some one-relator groups with torsion, J. Algebra 71 (1981), 132-140.

3. Residually finite one-relator groups with torsion, Arch. Math. (Basel) 37 (1981). 97-105.

4. S. M. Armstrong, One relator groups with non-trivial centre, M. Phil. Thesis, Queen Mary College, 1977.

5. B. Baumslag and F. Levin, A class of one-relator groups with torsion, Arch. Math. (Basel) 33 (1979), $209-215$.

6. G. Baumslag, Residually finite one-relator groups, Bull. Amer. Math. Soc. 73 (1967), 618-620.

7. G. Baumslag and D. Solitar, Some two-generator one-relator non-Hopfian groups, Bull. Amer. Math. Soc. 68 (1962), 199-201.

8. A. M. Brunner and R. G. Burns, Groups in which every finitely generated subgroup is almost a free factor, Canad. J. Math. 31 (1979), 1329-1338.

9. J. L. Dyer, Separating conjugates in amalgamated free products and HNN extensions, J. Austral. Math. Soc. Ser. A 29 (1980), 35-51. 
10. J. Fischer, A Karrass and D. Solitar, On one-relator groups having elements of finite order, Proc. Amer. Math. Soc. 33 (1972), 297-301.

11. S. Lipschutz, Generalization of Dehn's result on the conjugacy problem, Proc. Amer. Math. Soc. 17 (1966), 759-762.

12. W. Magnus, A. Karrass and D. Solitar, Combinatorial group theory, Wiley, New York, 1966.

13. A. W. Mostowski, On the decidability of some problems in special classes of groups, Fund. Math. 59 (1966), 123-135.

14. B. B. Newman, Some results on one-relator groups, Bull. Amer. Math. Soc. 74 (1968), 568-571.

15. P. Stebe, Conjugacy separability of certain free products with amalgamations, Trans. Amer. Math. Soc. 156 (1971), 119-129.

Department of Pure Mathematics, University of Waterloo, Waterloo, Ontario, Canada, N2L 3G1 